American Journal of Applied Sciences 6 (6): 1153-1157, 2009

ISSN 1546-9239

(C) 2009 Science Publications

\title{
Effect of Divalent Ions (A = Ca, Ba and Sr) Substitution in La-A-Mn-O Manganite on Structural, Magnetic and Electrical Transport Properties
}

\author{
K.P. Lim, S.W. Ng, S.A. Halim, S.K. Chen and J.K. Wong \\ Department of Physics, Faculty of Science, University Putra Malaysia, \\ 43400 UPM Serdang, Malaysia
}

\begin{abstract}
Problem statement: Microstructure of the grain will influence the properties of a polycrystalline manganites when different dopant is introduced. In this work, an effort had been made to investigate the influence of $\mathrm{Ca}, \mathrm{Ba}$ and $\mathrm{Sr}$ substitution in La site. Approach: Polycrystalline manganites compound of $\mathrm{La}_{0.67} \mathrm{~A}_{0.33} \mathrm{MnO}_{3}$ where $\mathrm{A}=\mathrm{Ba}, \mathrm{Sr}$ and $\mathrm{Ca}$ had been prepared via conventional solid-state reaction method. The structure, microstructure, magnetic and electrical properties had been investigated using XRD, SEM, VSM and four-point probe techniques. Results: XRD spectrums showed that LBMO and LCMO were in single-phase orthorhombic structure whereas LSMO was rhombohedral structure. Scanning electron micrographs showed that LSMO had smaller average grain size as compared to LBMO. However, for LCMO, there was no clear grain boundary that can be observed and all the grains were well connected. The difference in the microstructure image might be due to the variance A-site cation that differs in grain growth. The Curie temperature, $\mathrm{T}_{\mathrm{c}}$ of LBMO and LSMO was 343.0 and $363.5 \mathrm{~K}$, respectively. But the $\mathrm{T}_{\mathrm{c}}$ for LCMO was lower then $300 \mathrm{~K}$. The $\mathrm{T}_{\mathrm{p}}$ for LCMO was $215 \mathrm{~K}$ while it was near or above 300K for LBMO and LSMO. All samples showed extrinsic Magnetoresistance (MR) effect. Higher MR was observed well below $T_{c}$ or $T_{p}$ where MR value increased monotonically with the decrease of temperature. This was associated with the different grain and grain boundary formation when different cation was substituted. LCMO displayed the highest low-field MR value (-15.82\% at $0.2 \mathrm{~T}, 90 \mathrm{~K})$ and high-field MR $(-25.60 \%$ at $1 \mathrm{~T}, 300 \mathrm{~K})$. Conclusion: Hence, electrical and magnetic transition temperature $\left(T_{p}\right.$ and $\left.T_{c}\right)$ were affected by the average A-site cation radius.
\end{abstract}

Key words: Ceramic, low field magnetoresistance, magnetoresistance

\section{INTRODUCTION}

In recent year there has been a lot of research study in Colossal Magnetoresistance (CMR) effects in rareearth manganite perovskite with general formula $\mathrm{La}_{1}$ ${ }_{x} \mathrm{~A}_{\mathrm{x}} \mathrm{MnO}_{3}(\mathrm{~A}=\mathrm{Ba}, \mathrm{Sr}, \mathrm{Pb}$ and $\mathrm{Ca})$ due to their potential technology application. These compounds are $\mathrm{Mn}^{3+}$ rich and doping of divalent atoms introduces mixture valency of $\mathrm{Mn}^{3+}$ and $\mathrm{Mn}^{4+}$ ions plays a major role in Double Exchange (DE) ferromagnetic interaction coupled with metallic resistivity. Double exchange effect is an exchange of electrons from neighboring $\mathrm{Mn}^{3+}$ to $\mathrm{Mn}^{4+}$ ions through oxygen when their core spin are parallel and hopping is not favored when they are anti-parallel. However, it has been claimed that an additional mechanism, Jahn-Teller distortion (JT) could be responsible for the transport properties. The JahnTeller effects cause further degeneracy of the $e_{g}$ orbital of $\mathrm{Mn}^{3+}$ in $\mathrm{MnO}_{6}$ octahedral and resulting electrical transport via hopping effect ${ }^{[1,2]}$.

The Magnetoresistance (MR) percentage of MR defined as $\left[\left(\mathrm{R}_{\mathrm{H}}-\mathrm{R}_{\mathrm{o}}\right) / \mathrm{R}_{\mathrm{o}}\right]^{*} 100$ where $\mathrm{R}_{\mathrm{H}}$ is the resistance in the present of magnetic field and $R_{0}$ is the resistance at zero field. In general, CMR effects of polycrystalline ceramic bulk exhibit two classes of Magnetoresistance (MR), the intrinsic and extrinsic MR. The former is referred to intragrain effect where its MR shows a maximum near paramagnetic-ferromagnetic transition temperature $\left(\mathrm{T}_{\mathrm{c}}\right)$ and maximum electrical resistivity at metal-insulator transition temperature $\left(\mathrm{T}_{\mathrm{p}}\right)$. The latter is due to the intergrain effect where higher MR could be observed over a wider temperature range below $T_{c}$ and is characteristic of a Low-Field MR (LFMR), which is believed to be due to Spin-Polarized Tunneling (SPT) or Spin-Dependent Scattering (SDS) at grain boundary ${ }^{[3,4,5]}$.

Corresponding Author: K.P. Lim, Department of Physics, Faculty of Science, University Putra Malaysia, 43400 UPM Serdang, Selangor, Malaysia 
Recently, the effect of grain boundaries in the polycrystalline manganites has been studied intensively, where the grain's shape or microstructure will be changed with different preparation process $^{[6,7]}$ or dopant ${ }^{[7-9]}$. It is found that substitution of divalent atoms with variance atomic radius and preparation process will influence the magnetic properties and electrical properties of the system $^{[6,7]}$. Therefore, in this study, an effort has been made to investigate the influence of various divalent atoms substituted in $\mathrm{La}^{3+}$.

\section{MATERIALS AND MATHODS}

The samples were prepared using the conventional solid-state reaction method. Stoichiometric amounts of $\mathrm{La}_{2} \mathrm{O}_{3}, \mathrm{SrCO}_{3}, \mathrm{BaCO}_{3}, \mathrm{CaCO}_{3}$ and $\mathrm{MnO}_{3}$ powders with high purity $(>99.5 \%)$ were mixed, ground and then calcined for $12 \mathrm{~h}$ at $900^{\circ} \mathrm{C}$. The obtained powder was then reground, sieve and pressed into pellets and subsequently sintered in an open tube furnace at $1300^{\circ} \mathrm{C}$ for $24 \mathrm{~h}$. The structure of the samples was characterized by X-Ray Diffraction (XRD) using a $\mathrm{Cu}$ $\mathrm{K} \alpha$ radiation at room temperature. Scanning Electron Microscope (SEM) scans (LEO1455 VP-SEM) were carried out to investigate the surface microstructure. The magnetic transition temperatures $\left(T_{c}\right)$ were obtained from 303-493 K using Vibration Sample Magnetometer (VSM, LakeShore 7400). Magnetoresistance measurement, which is inserted in the liquid nitrogen cryostat, was used to measure the change in resistance under an external applied magnetic field up to 1 Tesla with temperature ranging from 90$300 \mathrm{~K}$. The electrical resistivity of all samples was measured by conventional four-point-probe technique in the temperature range $20-300 \mathrm{~K}$ and the data are collected in the heating mode.

\section{RESULTS}

Figure 1 shows the XRD spectrum before and after sintering process. Seven significant peaks that representing different hkl plane had been observed from all XRD spectrums.

Figure 2 shows the SEM images with 3000× magnification obtained at room temperature. Overall, uniform grain size with significant pores is formed for all samples. The average grain size for LBMO and LSMO are 1.0-2.5 and 0.5-2.0 $\mu \mathrm{m}$ respectively. However, for LCMO, all the grains were well connected to each other and no clear grain boundary can be observed.

The derivation of magnetization against temperature $\left(\mathrm{dm} \mathrm{dT}{ }^{-1}\right)$ versus temperature curve from 300-420 K shown in the Fig. 3. The results shown that $\mathrm{T}_{\mathrm{c}}$ for LBMO and LSMO systems were 343.0 and $363.5 \mathrm{~K}$, respectively.

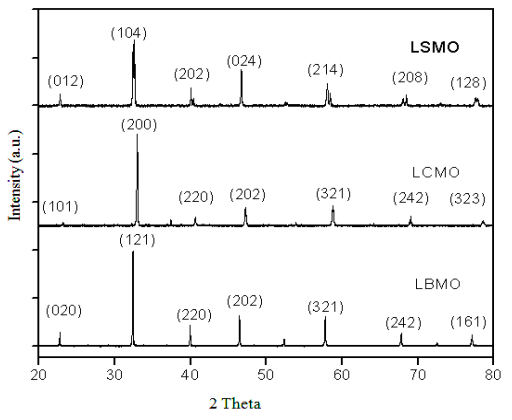

Fig. 1: XRD pattern for LBMO, LCMO and LSMO after sintering at $1300^{\circ} \mathrm{C}$

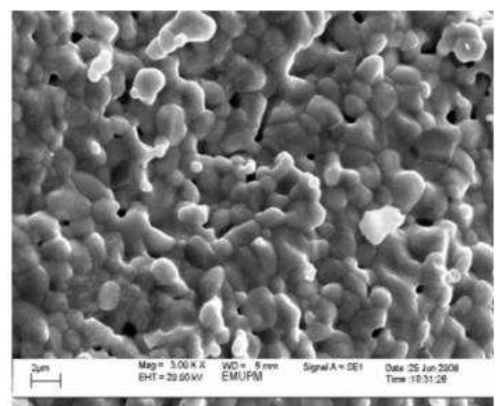

(a)

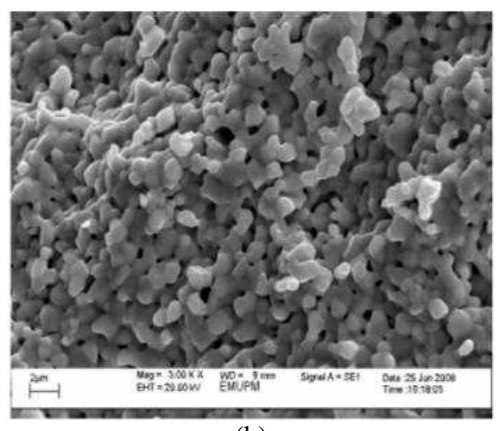

(b)

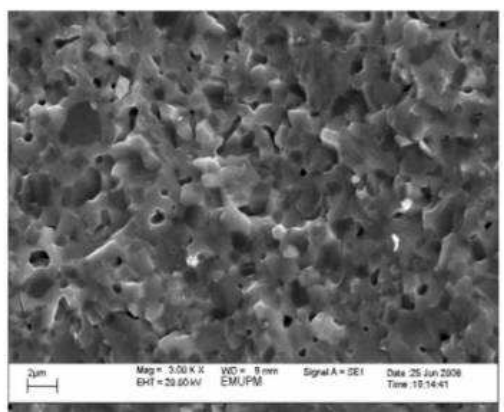

(c)

Fig. 2: SEM images for (a): LBMO, (b): LSMO and (c): LCMO after sintering at $1300^{\circ} \mathrm{C}$ 


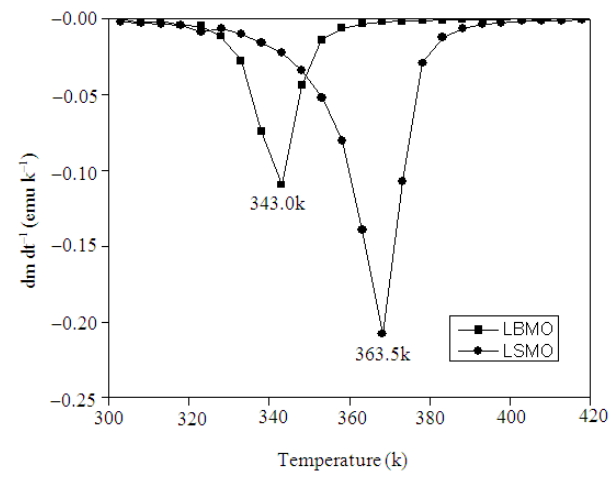

Fig. 3: The derivation of magnetization $\left(\mathrm{dm} \mathrm{dT}^{-1}\right)$ against temperature curve for LBMO

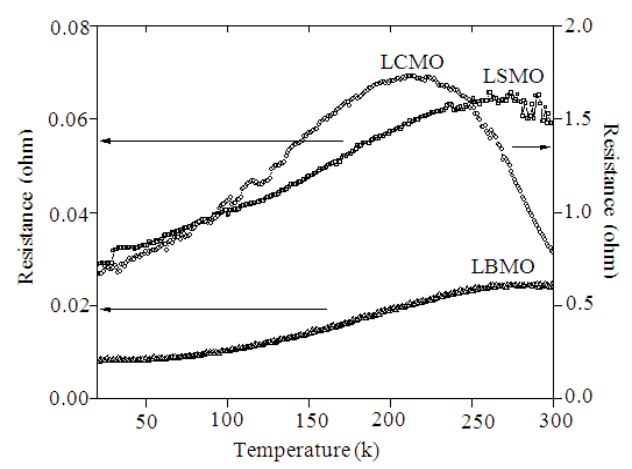

Fig. 4: Resistance Vs temperature curve for LBMO, LSMO and LCMO

The temperature dependence of the resistance for LBMO, LSMO and LCMO were shown in Fig. 4. The results shown that the resistance of LCMO sample increases when temperature drops from 300-215 K and its resistance decreases when temperature further drops to $80 \mathrm{~K}$. The resistance reach maximum value at temperature around $215 \mathrm{~K}$. However, for LSMO and LBMO samples, their resistance showed a decrease with the drop of temperature.

The percentage of MR curve of all the samples have been measured from 90-300 K with an external magnetic field up to 1 Tesla as shown in Fig. 5. With an increase of the magnetic field, the resistance for all samples has found to be decreased. All there samples shown a negative value of MR and no saturation had been observed. The MR curves gave two significant different regions where faster drop of MR value at $0-0.1 \mathrm{~T}$ follow by a slower drop at $0.1 \mathrm{~T}-1.0 \mathrm{~T}$. LCMO have highest $-15.8 \%$ LFMR value $(0.2 \mathrm{~T}$ at $90 \mathrm{~K})$ followed by LBMO and LSMO systems as $-12.7 \%$ and $-12.3 \%(0.1 \mathrm{~T}$ at $90 \mathrm{~K})$, respectively. The highest CMR value is found in LCMO system $(-25.6 \%)$ followed by LBMO system $(-17.6 \%)$ and LSMO system (-17.3\%) at 90K with 1 Tesla.

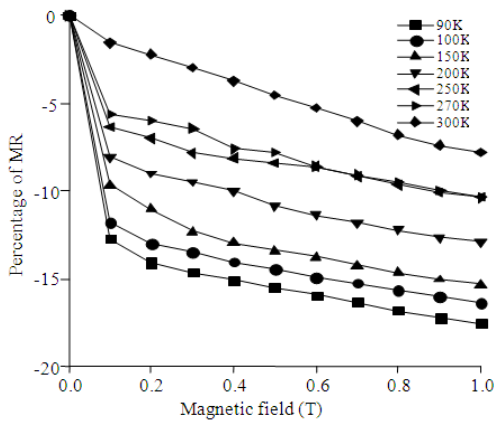

(a)

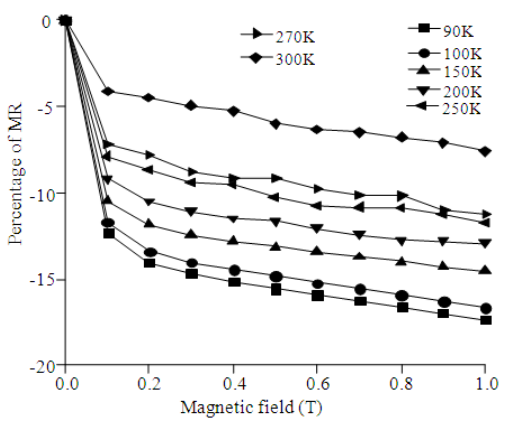

(b)

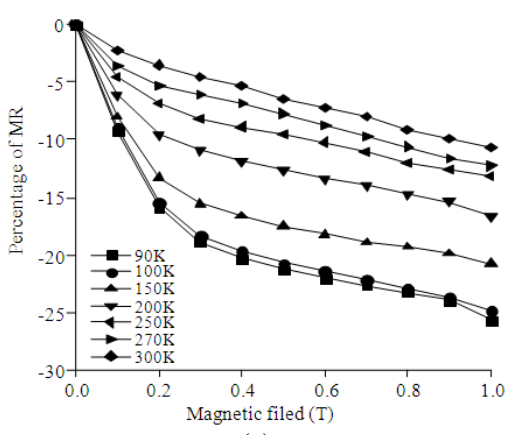

(c)

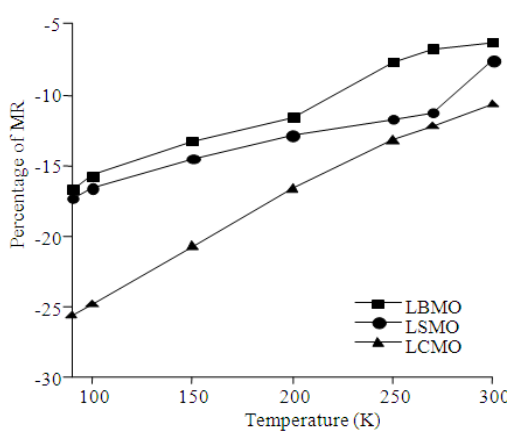

(d)

Fig. 5: Field dependence of MR curve at different temperature for (a): LBMO (b): LSMO (c): LCMO (d): Temperature dependence of the MR ratio in 1 Tesla field 
Figure 5d shows the MR value versus temperature for all samples of 1 Tesla. The MR value increases monotonically with the decrease of temperature.

\section{DISCUSSION}

XRD results obtained from Fig. 1 shows that, after sintering at $1300^{\circ} \mathrm{C}$ for $24 \mathrm{~h}$, the bulk samples have completely formed into a polycrystalline single phase compound where no significant extra peak is observed. Comparing to the standard ICDD (references code 01089-0569, 00-049-0416 and 00-050-0308 for $\mathrm{La}_{0.67} \mathrm{Ba}_{0.33} \mathrm{MnO}_{3}$ (LBMO), $\mathrm{La}_{0.67} \mathrm{Sr}_{0.33} \mathrm{MnO}_{3}$ (LSMO) and $\mathrm{La}_{0.67} \mathrm{Ca}_{0.33} \mathrm{MnO}_{3}$ (LCMO) respectively), LBMO and LCMO showed orthorhombic system whereas LSMO showed rhombohedral system which agree with the early report ${ }^{[7,10]}$.

Different microstructures as shown in Fig. 2 were observed for different sample when viewed under SEM. For LCMO system, microstructural densification has taken place where grain diffusion occurred during sintering at $1300^{\circ} \mathrm{C}$. This causes the formation of meltlike form structure where no clear grain boundary can be identified and thick disorder layer might have occurred to connect the grains together. The variance of surface microstructure grain growth might be due to the different activation energy of $\mathrm{Ba}^{2+}, \mathrm{Sr}^{2+}$ and $\mathrm{Ca}^{2+}$ for diffusion reaction to take place during sintering process. This variation is also obtained in different preparation method with similar divalent substitution in previous reports ${ }^{[9,10]}$.

Magnetic properties (Fig. 3) investigation via VSM measurement shown that LBMO and LSMO systems show a transition from ferromagnetic to paramagnetic (Curie temperature, $\mathrm{T}_{\mathrm{C}}$ ) at 343.0 and $363.5 \mathrm{~K}$, respectively. This result is slightly higher than the reported value ${ }^{[9]}$. From previous study, $\mathrm{T}_{\mathrm{c}}$ for LCMO is $240 \mathrm{~K}$, which is lower than the measurement range $(300 \mathrm{~K})^{[10]}$. Hence, $\mathrm{T}_{\mathrm{C}}$ was unable to identify for this sample.

The electrical properties (Fig. 4) shown that a $T_{p}$ transition at $215 \mathrm{~K}$ was obtained in LCMO sample. It exhibits metallic behavior below $T_{p}$ and a semiconductor- like behavior above $T_{p}$ where maximum resistance occurred at $T_{p}$. However, LBMO and LSMO do not show a clear $T_{p}$ transition over the measurement range indicating that $T_{p}$ might be around or above $300 \mathrm{~K}$. Below $300 \mathrm{~K}$, all samples show metallic behavior where their resistance decreases monotonically with the drop of temperature. Below $\mathrm{T}_{\mathrm{p}}$, the $\mathrm{Mn}^{3+}-\mathrm{O}^{2-}-\mathrm{Mn}^{4+}$ coupling produce conduction through charge transfer from halffilled to empty $e_{g}$ orbital due to double exchange effect and this effect is more favored when they are aligned parallel. Thus electron transport is easier and its resistance decreases.

As compared to the Curie temperature, $\mathrm{T}_{\mathrm{p}}$ is lower than $T_{c}$. This might be due to the occurrence of the antiferromagnetic or disordered insulating region at the grain boundary. For single crystalline compound, $T_{p}$ and $T_{c}$ are normally quite closed ${ }^{[11]}$. However, in polycrystalline samples, these disordered layers at the grain boundary influenced the electron transport and weakened the DE mechanism. However, this insulating disordered region does not strongly affect the magnetic property. So, $T_{p}$ is shifted faster to lower value ${ }^{[12]}$.

Negative MR value had been observed as shown in Fig. 5. By applying an external magnetic field, the localized $t_{2 g}$ spin will be aligned parallel to each other. This enhanced $\mathrm{e}_{\mathrm{g}}$ electrons mobility or DE mechanism between $\mathrm{Mn}^{3+}-\mathrm{O}-\mathrm{Mn}^{4+}$. Hence, the resistance dropped when magnetic field was applied and negative MR was obtained. All three systems have not achieved saturation with a magnetic field up to 1 Tesla, meaning that higher magnetic field is needed to fully align all localized spin. This might be due to the existing of some disordered region at the grain boundary. The samples give two significant different regions or gradient, which is known as Low Field Magnetoresistance (LFMR) effect (0-0.1T for LBMO and LSMO systems and 0-0.2T for LCMO system) followed by a linear drop of resistance so called intrinsic MR effect. The LFMR behavior is commonly shown in the polycrystalline form and believes to be caused by the spin dependent scattering and spin dependent tunneling effect at the interfaces of grain boundary ${ }^{[5]}$.

From Fig. 5, the highest \%MR value is not observed near $T_{p}$ or $T_{c}$ and the MR value decreases as the temperature increases. This result is typical for polycrystalline compound that shows extrinsic MR effect where higher MR is observed well below $T_{c}$ or $T_{p}{ }^{[13,14]}$.

\section{CONCLUSION}

In summary, polycrystalline $\mathrm{La}_{0.67} \mathrm{~A}_{0.33} \mathrm{MnO}_{3}$ (where $\mathrm{A}=\mathrm{Ba}, \mathrm{Ca}$ and $\mathrm{Sr}$ ) have been prepared by solid-state reaction method and their structure, magnetic, electrical and MR effect in bulk polycrystalline are investigated. Different of A-site doping elements give different electrical and magnetic properties. From XRD analysis, LBMO and LCMO showed orthorhombic system whereas LSMO showed rhombohedral system. Grain formation varied with the A-site doping. Smaller A-site cation radius promotes grain growth. Curie temperature for LBMO and LSMO are 343.0 and $363.5 \mathrm{~K}$, respectively. All systems have 
relatively lower $T_{P}$ as compared to Curie temperature, mainly due to the grain boundaries disordered layer effect. LCMO system has the highest CMR value of $25.6 \%$ at $90 \mathrm{~K}$ with 1 Tesla. The highest LFMR effect at $0.2 \mathrm{~T}$ is observed in LCMO system $(-15.8 \%$, at $90 \mathrm{~K})$.

\section{ACKNOWLEDGEMENT}

The Ministry of Science, Technology and Innovation of Malaysia is gratefully acknowledged for the grant under Science Fund vote: 03-01-04-SF0088 (Fabrication of Multilayers Manganite Thin Film having LFMR Effect Using Pulsed Laser Ablation Technique) and 03-01-04-SF0009 (Study of Novel Nanoscale multiferroic via magnetic and electrical properties).

\section{REFERENCES}

1. Rama, N., V. Sankaranarayan and M.S. Ramachandra Rao, 2008. Electrical resistivity, thermoelectric power and electron spin resonancestudies on $\operatorname{Pr}(1-\mathrm{x}) \operatorname{SrxMnO} 3$ ( $\mathrm{x}=0.2,0.26$ and 0.3). J. Alloys Comp., 466: 12-16. DOI: 10.1016/j.jallcom.2007.11.046

2. Dagotto, E., T. Hotta and A. Moreo, 2001. Colossal magnetoresistant materials: The key role of phase separation. Phys. Rep., 344: 1-153.

3. Ravi, V., S.D. Kulkarni, V. Samuel, S.N. Kale, J. Mona, R. Rajgopal, A. Daundkar, P.S. Lahoti and R.S. Joshee, 2007. Synthesis of $\mathrm{La}_{0.7} \mathrm{Sr}_{0.3} \mathrm{MnO}_{3}$ at $800^{\circ} \mathrm{C}$ using citrate gel method. Ceramics Int., 33: 1129-1132. DOI: 10.1016/j.ceramint.2006.02.008

4. Kameli, P., H. Salamati and A. Aezami, 2008. Influence of grain size on magnetic and transport properties of polycrystalline $\mathrm{La} 0.8 \mathrm{Sr} 0.2 \mathrm{MnO} 3$ manganites. J. Alloys Comp., 450: 7-11. DOI: 10.1016/j.jallcom.2006.10.078

5. Zhao, L. F., W. Chen, J.L. Shang, Y.Q. Wang, G.Q. Yu, X. Xiao, J.H. Miao, Z.C. Xia and S.L. Yuan, 2006. Low magnetoresistance obserced in polycrystalline $\mathrm{La}_{0.67} \mathrm{Ca}_{0.33 \mathrm{Mn} 1-x} \mathrm{O}_{3}$ sintered at low temperature", Mater. Sci. Eng. B., 127: 193-197. DOI: 10.1010/j.mseb.2005.10.020

6. Wang, T., X. Fang, W. Dong, R. Tao, Z. Deng, D. Li, Y. Zhao, G. Meng, S. Zhou and X. Zhu, 2008. Mechanochemical effects on microstructure and transport properties of nanocrystalline La0.8Na0.2MnO3 ceramics. J. Alloys Comp., 458: 248-252. DOI: 10.1016/j.jallcom.2007.04.023
7. Kalyana Lakshmi, Y., G. Venkataiah, M. Vithal and P. Venugopal Reddy, 2008. Magnetic and electrical behavior of $\mathrm{La}_{1-\mathrm{x}} \mathrm{A}_{\mathrm{x}} \mathrm{MnO}_{3}(\mathrm{~A}=\mathrm{Li}, \mathrm{Na}, \mathrm{K}$ and $\mathrm{Rb}$ ) manganites. Phys. B., 403: 3059-3066. DOI: 10.1016/j.physb.2008.03.018

8. Venkataiah, G., V. Prasad and P. Venugopal Reddy, 2007. Influence of A-site cation mismatch on structural, magnetic and electrical properties of lanthanum manganites. J. Alloys Comp., 429: 1-9. DOI: 10.1016/j.jallcom.2006.03.081

9. Im, H.S., G.B. Chon, S.M. Lee, B.H. Koo, C.G. Lee and M.H. Jung, 2007. Preparation and characterization of $\mathrm{La}_{0.7} \mathrm{AE}_{0.3} \mathrm{MnO}_{3}(\mathrm{AE}=\mathrm{Ca}, \mathrm{Sr}$, $\mathrm{Ba}$ ): Perovskite structure manganites. J. Magnet. Magnet. Mater., 310: 2668-2670. DOI: 10.1016/j.jmmm.2006.12.002

10. Vavra, F.I., F. Gomory, J. Souc, J. Bydzovsky, P. Kovac, J. Dobrovodsky and M. Marysko, Microstructuredependent magnetoresistance in $\mathrm{La}_{1-x} \mathrm{MnO} 3$ thin flims. J. Magnet. Magnet. Mater., 211: 6772.

11. Venkataiah, G. and P.V. Reddy, 2005. Structural, magnetic and magnetotransport behavior of some Nd-based perovskite manganites. Solid State Commun., 136: 114-119. DOI: 10.1016/j.ssc.2005.04.014

12. Lisboa-Filho, P.N., A.W. Mombru, H. Pardo, E.R. Leite and W.A. Ortiz, 2004. Extrinsic properties of colossal magnetoresistance samples. Solid State Commun., 130: 31-36. DOI: 10.1016/j.ssc.2004.01.024

13. Lei, L.W., Z.Y. Fu and J.Y. Zhang, 2006. Influence of sintering temperature on microstructure and magnetotranspot properties of $\mathrm{La}_{0.8} \mathrm{Na}_{0.2} \mathrm{MnO}_{3}$. Mater. Lett., 60: 970. DOI: 10.1016/j.matlet.2005.10.060

14. Xiong, Y.H., W. Xu, Y.T. Mai, H.L. Pi, C.L. Sun, X.C. Bao, W.H. Huang and C.S. Xiong, 2008. The microstructure and electronic transport properties of mechanical milled $\mathrm{La}_{2 / 3} \mathrm{Ca}_{1 / 3} \mathrm{MnO}_{3}$ perovskites. $\mathrm{J}$. Magnet. Magnet. Mater., 257-262. DOI: 10.1016/j.jmmm.2007.06.013 